\title{
Gelfoam-induced Swallowing Difficulty after Anterior Cervical Spine Surgery
}

\author{
Joo Chul Yang, Tae Wan Kim, Kwan Ho Park \\ Department of Neurosurgery, VHS Medical Center, Seoul, Republic of Korea
}

Symptomatic diffuse idiopathic skeletal hyperostosis (DISH) is not common. Gelfoam is one of the most commonly used topical hemostatic agents. But, in the partially moistened state, air retained in its pores may result in excessive expansion on contact with liquid. The onset of swallowing difficulty after anterior cervical spine surgery due to appling gelfoam is a rare complication. A 77-year-old man with swallowing difficulty was admitted to our hospital and we diagnosed him as DISH confirmed by radiological study. After removing the DISH, patient's symptom was relieved gradually. However, on postoperative day (POD) 7, the symptom recurred but lesser than the preoperative state. We confirmed no hematoma and esophageal perforation on the operation site. We observed him closely and controlled the diet. Three months later, he had no symptom of swallowing difficulty, and was able to be back on a regular diet, including solid foods. We present a complication case of swallowing difficulty occurring by gelfoam application.

Key Words: Diffuse idiopathic skeletal hyperostosis $\cdot$ Swallowing difficulty $\cdot$ Gelfoam

\section{INTRODUCTION}

Cervical anterior osteophytes accompanying diffuse idiopathic skeletal hyperostosis (DISH) are most frequently asymptomatic. Most cases are treated conservatively. However, large osteophytes will occur with symptoms that include swallowing difficulty. In these cases, surgical management will be needed. Gelfoam is widely used in spine surgery and its complication is rare. Gelfoam has been used to control the bleeding and prevent scar adhesion when used after laminectomy as an effective interposing membrane ${ }^{8)}$. However, it has the potential to compress the adjacent structure and change to the hard epidural fibrosis, which may be overlooked. Here, we report on the gelfoam induced swallowing difficulty, including free air formation and swelling after anterior cervical spine surgery.

\footnotetext{
- Received: March 8, 2013 • Revised: May 23, 2013

- Accepted: June 3, 2013

Corresponding Author: Tae Wan Kim, MD

Department of Neurosurgery, VHS medical center, 53 Jinhwangdo-ro,

61-gil, Gangdong-gu, Seoul 134-791, Republic of Korea

Tel: +82-2-2225-1363, Fax: +82-2-2225-4152

E-mail: euro3399@naver.com
}

\section{CASE REPORT}

A 77-year-old man was presented with neck pain for several months. Cervical lateral radiographics demonstrated a multisegmental anterior osteophyte formation, especially at C 3-4 (Fig 1-A). However, he did notcomplain of any respiratory and swallowing difficulties. Therefore, we decided that conservative treatment may be better suited for this case. Five months later, he was admitted to our hospital with swallowing difficulty, which occurred ten days prior to presentation. He could only eat soft foods with frequent coughing. A computed tomography (CT) scan was performed and demonstrated anterior osteophyte with indentation on the esophagus (Fig 1-B). Magnetic resonance (MR) images showed multisegmental anteriorly flowing osteophyte formation with esophageal indentation. The videofluoroscopic swallowing study (VFSS) showed abnormal coordination of peristaltic movement and reflux to the trachea on a pharyngeal phase. We recommended tube feeding.

We removed the DISH via anterolateral cervical approach and applied gelfoam on the osteophyte removal site, owing to the uneven surface of the remained osteophyte. After the operation, the patient was relieved from the swallowing difficulty and had normal diet, except solid foods. On POD 7, he complained of swallowing difficulty again, but the degree 

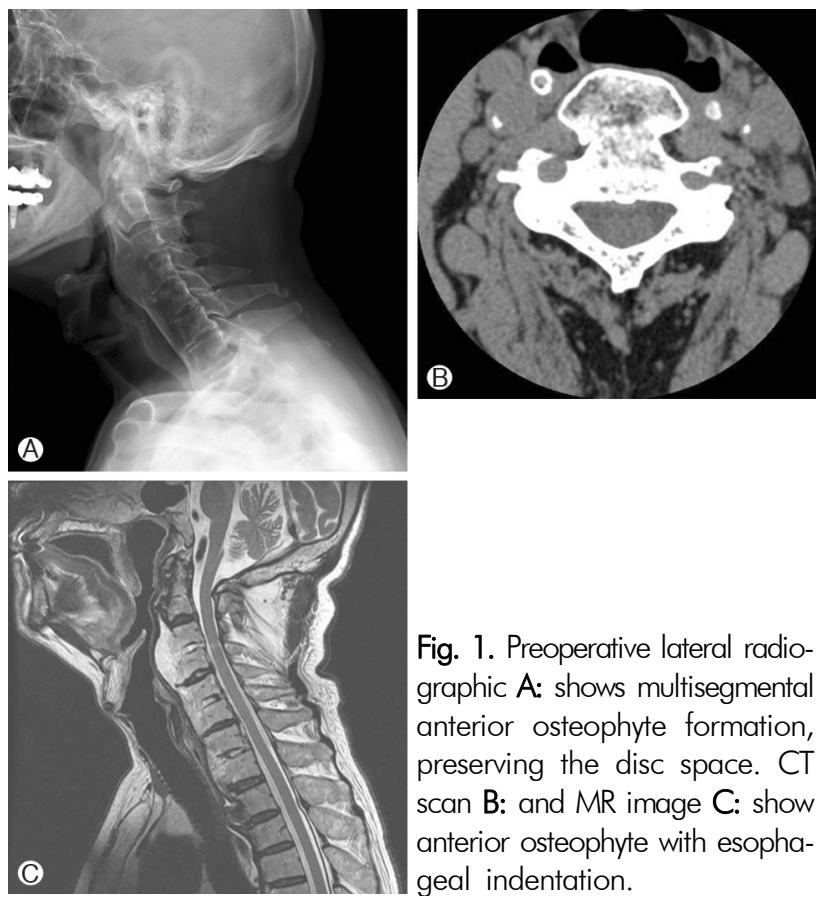

Fig. 1. Preoperative lateral radiographic A: shows multisegmental anterior osteophyte formation, preserving the disc space. CT scan B: and MR image C: show anterior osteophyte with esophageal indentation.

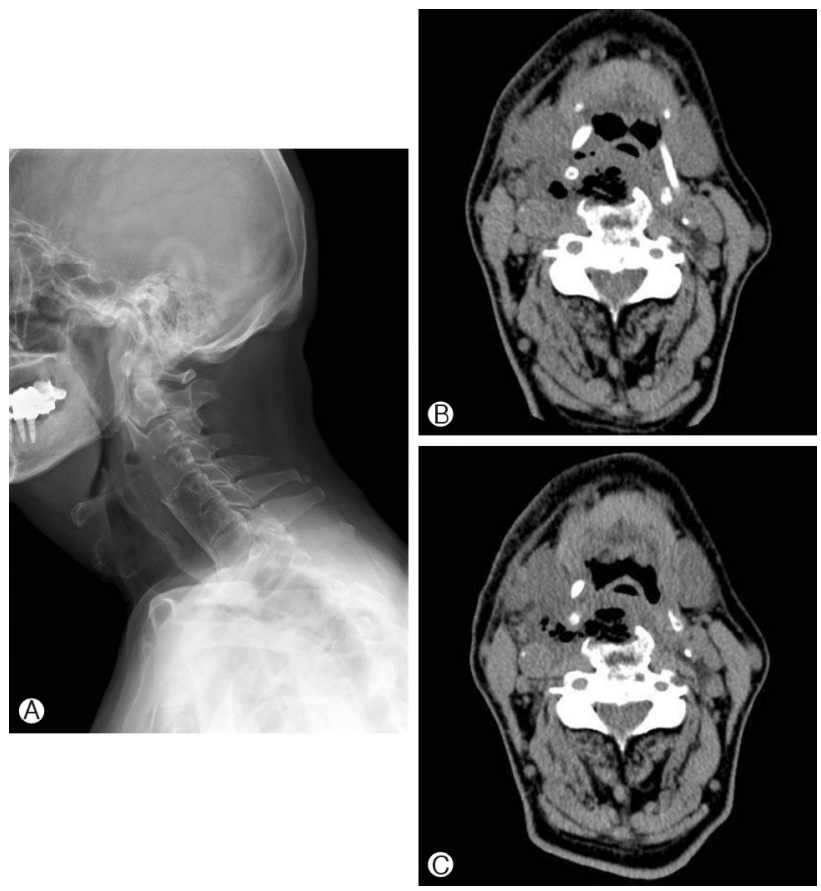

Fig. 2. Postoperative lateral radiographic $A$ : and $C T$ scan $B$ : show free air formation on the operative site and slight displacement of the esophagus. Follow up CT scan C: shows lesser compression to the esophagus, in spite of the free air and swelling that still remain, 14 days later.

of dysphagia was less than the preoperative state. His vital signs, including respiration, were stable. Cervical lateral radiographics demonstrated air formation on osteophyte removal

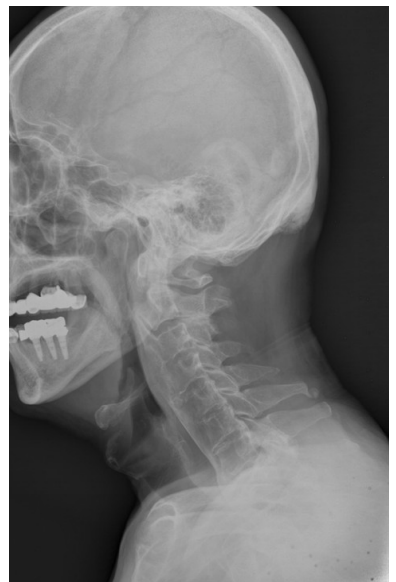

Fig. 3. Lateral radiographic shows no swelling and free air, 3 months later.

site (Fig 2-A). CT scan demonstrated free air with low density fluid collection on the prevertebral and right paravertebral space and swelling (Fig 2-B). On laryngoscopic examination, the perforation and fistula were not noted. Endoscopic examination showed no esophageal perforation. The repeated VFSS showed a decline of laryngeal elevation and disability of laryngeal closure, and recommended small amounts oral feeding. The symptom was not severe and respiratory difficulty was not noted. We closely observed the patient.

Fourteen days later, the swallowing difficulty was relieved and repeated CT scan showed lesser compression to the esophagus, in spite of the free air and swelling that still remain (Fig 2-C).

Three months later, the patient could eat all foods, including solid foods. The radiographic showed no swelling and free air (Fig 3).

\section{DISCUSSION}

Most of the anterior cervical osteophytes may be asymptomatic. DISH is a degenerative disorder of unknown cause and is a rare cause of dysphagia. For most patients, conservative treatment is suitable. Initial treatment for dysphagia includes muscle relaxants, antireflux medications, steroids, diet control, and postural changes when swallowing.

DISH and ankylosing spondylitis are the most common causes of bony outgrowth of the cervical spine, causing dysphagia and aspiration in the elderly ${ }^{5,9}$. However, with depending their size and location, symptoms such as dysphagia or dyspnea, may occur. Dysphagia due to osteophytes may be the result of these mechanism: direct compression of the pharynx or esophagus, inflammatory reaction of soft tissue around the esophagus, disturbances of normal epiglottis tilt over the laryngeal inlet by the osteophytes at the $\mathrm{C} 3-\mathrm{C} 4$ level $^{2,10)}$. Once the symptoms develop, osteophytectomy will be the choice 
of treatment. Osteophytecomy is reserved for patients with persistent symptoms, despite optimal medical management and coinciding radiographic evidence, suggesting functional impairment of swallowing ${ }^{4,11}$.

Postoperative dysphagia, hematoma, and recurrent laryngeal nerve palsy are the most common complications. Amongst all of them, postoperative dysphagia is the most common complication $^{7)}$, although dysphagia is a subjective symptom. Postoperative soft tissue swelling, hematoma, and adhesion formation around the device will be the cause. Retraction of the pharynx and esophagus during surgery may lead to edema. Disruption of the vagus nerve or pharyngeal plexus is another possible etiology ${ }^{12)}$.

Long-term complication on DISH patients with dysphagia are at great risk for recurrence of osteophytes, following surgical resection. The surgeons should follow the patients for more than 10 years, postoperately ${ }^{10)}$.

Various topical hemostatic agents are used to control intraoperative and postoperative bleeding. Gelfoam is one of the most commonly used topical hemostatic agents. Gelfoam has a hemostatic and absorptive nature. The immunogenicity of gelfoam or microfibrillar collagen may promote postoperative swelling ${ }^{6}$. In the partially moistened state, air retained in its pores may result in excessive expansion on contact with liquid $^{8)}$. On MR images, the inhomogeneous signal on T1- and T2-weighted images is consistent with a mixture of fluid, blood and gelfoam, after postoperative 10 days and curvilinear isointense layer with circumscribed, predominantly T2 hyperintense collection after 3 weeks ${ }^{6}$.

Gelfoam may absorb blood and become surrounded with a clot for as long as 3 days. The maximum tissue response to its presence usually occurs by postoperative day $12^{8)}$. Squeezing the gelfoams to expel air bubble before use is recommended to prevent excessive engorgement ${ }^{8}$.

The application of gelfoam around the spinal cord may compress the spinal cord ${ }^{1,3,6}$. Expansion of the gelfoam with blood and obstruction of any draining area is likely the cause. Large pieces of gelfoam should be removed, once hemostatic control is accomplished ${ }^{1)}$. In our case, the used gelfoam is somewhat larger and should not be squeezed fully before using.

\section{CONCLUSION}

The authors present a case of gelfoam-induced swallowing difficulty after anterior cervical surgery. Although the use of gelfoam is relatively safe, for precautious measures, we should avoid using gelfoam that are large in size and should handle it with proper care. The gelfoam-induced complication, which not includes neural tissue compromise and respiratory difficulty, may be well treated conservatively.

\section{REFERENCES}

1. Alaxander DH, Stauffer ES: Gelfoam-induced acute quadriparesis after cervical decompression and fusion. Spine (Phila $\mathrm{Pa}$ ) 20(8): 970-971, 1995

2. Aydin E, Akdogan V, Akkuzu B, Kirbas I, Ozgirgin ON: Six cases of Forestier syndrome, a rare cause of dysphagia. Acta Otolaryngol 21:259-263, 2006

3. Buchowski JM, Briedwell KH, Lenke LG, Good CR: Epidural spinal cord compression with neurologic deficit associated with intrapedicular application of hemostatic gelatin matrix during pedicle screw insertion. Spine (Phila Pa) 34(13):473-477, 2009

4. Carlson ML, Archibald DJ, Graner DE, Kasperbauer JL: Surgical management of dysphagia and airway obstruction in patients with prominent ventral cervical osteophytes. Dysphagia 26:34-40, 2011

5. De Jesus-Monge WE, Cruz-cuevas EI: Dysphagia and lung aspiration secondary to anterior cervical osteophytes: A case report and review of the literature. Ethn Dis 18:137-140, 2008

6. Epstein NE, Silvergleid RS, Hollingsworth R: Increased postoperative cervical myelopathy and cord compression resulting from the use of gelfoam. Spine J 9:19-21, 2009

7. Fountas KN, Kapsalaki EZ, Nikolakakos LG, Smisson HF, Johnston KW, Grigorian AA, et al: Anterior cervical discectomy and fusion associated complications. Spine (Phila Pa) 32(21):23102317, 2007

8. Friedman J, Whitecloud TS $3^{\text {rd }}$ : Lumbar cauda eqina syndrome associated with the use of gelfoam. Spine (Phila Pa) 26(20):485487, 2001

9. Krishnarasa B, Vivekanandarajah A, Ripoll L, Chang E, Wetz R: Diffuse idiopathic skeletal hyperostosis (DISH) - A rare etiology of dysphagia. Clin Med Insights Arthritis Musculoskelet Disord 4:71-75, 2011

10. Miyamoto K, Sugiyama S, Hosoe H, linuma N, Suzuki Y, Shimizu $\mathrm{K}$ : Postsurgical recurrence of osteophytes causing dysphagia in patients with diffuse idiopathic skeletal hyperostosis. Eur Spine J 18:1652-1658, 2009

11. Song BK, Eun JP, Park SS, Kim SH: Dysphagia caused by ossification of the cervical anterior longitudinal ligament. Report of two cases. Kor J Spine 6(2):86-89, 2009

12. Winslow CP, Meyers AD: Otolaryngologic complications of the anterior approach to the cervical spine. Am J Otolaryngol 20: 16-27, 1999 